\section{Effects of Low Level Laser Therapy on Orthodontic Tooth Movement: A Systematic Review}

\section{Abstract}

The objective of this systematic review is to extract and study evidences available toassess the effectiveness of low level laser therapy (LLLT) on acceleration of Orthodontic tooth movement. The data sources used were electronic data bases including EMBASE, PubMed, Scopus and Google Scholar. References from collectedarticles were hand searched. Search was not bound with any time limits. Randomized control and clinical trials were also included. Search terms included were orthodontic movement speed/accelerated tooth movement/ LLLT/ Coldsoft diode laser/ Ga-Al-As laser/ low intensity laser/ light therapy/ phototherapy/ photobiostimulation. Only those studies which falls in the moderate to high category on assessment with Cericato et al. method for bias assessment were included. Double extractors did the data collection and at the time of controversies a blinded expert were approached for final decision. Nine studies seemed to be eligible for this study. An energy input of 4.2-8J sq.cm, wavelength of 780-904 nm applied for 80-100 sec/tooth within a time period of 4-6 days per month was found to be effective in demonstrating accelerated orthodontic tooth movement. Some scientific evidence for low level laser therapy causing accelerated orthodontic tooth movement was demonstrated. Those high evidence studies which came up with laser having no benefits on tooth movement explained their pit falls in the discussion, giving reason why they failed to observe any benefit in tooth movement.

Keywords: RCT; LLLT; Accelerated orthodontic tooth movement; Laser; Biostimutlation; Minimal invasive accelerated tooth movement

\section{Teena Maria Wilson* and Sandhya Jain \\ Department of Orthodontics and Dentofacial Orthopedics, Government College of Dentistry, Indore, Madhya Pradesh, India}

\section{*Corresponding author: \\ Dr. Teena Maria Wilson \\ tinamariawills@gmail.com}

\section{Department of Orthodontics and} Dentofacial Orthopedics, Govt. College of Dentistry, Indore, Madhya Pradesh, India.

Tel: 9447541371

Citation: Wilson TM, Jain S (2018) Effects of Low Level Laser Therapy on Orthodontic Tooth Movement: A Systematic Review. J Orthod Endod Vol.4 No.4:14

\section{Introduction}

Orthodontia is not that well known for treatment duration as treatment time lasts for one year and a half when fixed appliances are used to treat moderate to severe cases of malocclusion [1]. But the efforts taken for developing and incorporating interventions into treatment for reducing treatment duration is a new ray of hope. Reduced treatment time is adventitious not only for patient's aesthetic and comfort concerns but also avoids the chances for root resorption, periodontal and gingival issues, alveolar bone resorption and caries susceptibility due to longer treatment period [2]. Even though hard tissue alveolar bone and periodontal ligament are in dynamic state of remodeling by combined action of fibroblasts, osteoclasts and osteoblasts, the patients are unaware about this complex condition and ask for faster treatment and this tendency is increasing $[3,4]$. Literature history have a variety of interventions including surgically assisted corticotomy [5], periodontal ligament distraction, dentoalveolar distractions, piezocision [6], surgically stimulated platelet rich plasma injection [7], bio mechanical self-ligating brackets [8], mechanically stimulating direct electric current, enzymatic micro battery, endogenous piezo electricity, LLLT, pulsed electromagnetic field, pharmacological approaches like injection of prostaglandin, 1,25 di hydroxyl cholecalciferol, corticosteroid hormones, parathyroid hormone, thyroxin [7] and relaxin injections [9] and as latest, nanotechnology with application of dry lubricants, polysulfone brackets, nanobio cells and LIPUS(low intensity pulsed ultra sound) [10]. Current evidences points to the effects of LLLT in orthodontic field as reduced post adjustment pain, increased bone formation at mid palatal sutural 
area following rapid palatal expansion and increase in the mini implant stability [11]. LLLT is able to change RANK (Receptor activator of nuclear factor kappa-B)/RANKL(Receptor activator of nuclear factor kappa-B ligand)/OPG (Osteoprotegrin) enzymatic system. Application of LLL causes an increase in the level of RANKL and RANKL/OPG ratio leading to increased bone turn over and there by accelerated tooth movement [11]. Considering the clinical practicability and patient acceptance LLLT had become prime choice of interest. The accelerating effect along with elevated bone remodelling, collagen synthesis, nitrate and nitrite turn over makes LLLT an important one among the minimally invasive procedures for acceleration of tooth movement [12]. For providing a precise view for readers on controversial relation between laser therapy and orthodontic tooth movement this systematic review was conducted with randomised control and clinical trials which experimented on the effect of low level laser treatment on orthodontic tooth movement.

\section{Materials and Methodology}

\section{Protocol}

This systematic review was conducted following guidelines of PRISMA [13] and guideline for systematic reviews [14].

\section{Objective and guiding question}

Objective was to assess the effect of LLLT on orthodontic tooth movement in comparison with control group. Based on PICOS (patient, intervention, comparison, outcome, study design) strategy [13] a guiding questions was formulated.

Patient - who requires orthodontic treatment?

Intervention - Orthodontic tooth movement with adjunctive LLLT

Comparison - Similar group /quadrant with same intervention but lacks LLLT

\section{Outcome - LLLT has an effect on tooth movement}

Study design - RCT(randomized control trial) or CCT (case control trial)with blinding

Guiding question formulated was, what is the effect of low level laser therapy in accelerated orthodontic tooth movement when compared with controls

\section{Inclusion criteria}

1. RCT's and CCT's which evaluated or reported the results or treatment parameters associated with accelerated orthodontic tooth movement with LLLT.

2. Orthodontic treatment by canine retraction for space closure.

3. Studies with subjects assigned either as control/placebo and experimental groups.

4. The outcome results should clearly mention whether success achieved or not. It can be either measurable variable of distance moved by tooth or speed of movement or rate of tooth movement or $\mathrm{p}$ value or a statement of result.

\section{Exclusion criteria}

1. Non randomized trials

2. Animal studies

3. Sample size less than 10

4. Articles, reviews, case reports, opinions, columns in publications,, letters, abstracts and pilot study

5. Publication language other than English.

6. Any studies which used other interventions along with LLLT (E.g. LLLT after corticision)

\section{Information source and search strategy}

Based on PRISMA guidelines an electronic search was conducted on four major databases Google scholar, Pub Med, EMBASE and Scopus. Combination of different key words was used for finding relevant studies. Last search was done on 12 JUNE 2018. References of major reviews were used for manual search in order to avoid missing out of any relevant studies while electronic searching. The key words used were orthodontic movement speed/accelerated tooth movement/ LLLT/ cold-soft diode laser/ Ga-Al-As laser/ low intensity laser/light therapy/phototherapy/ photo bio stimulation.

\section{Literature flow}

The literature search done is given as Figure 1. Out of four electronic databases, a total of 342 articles were evaluated. From

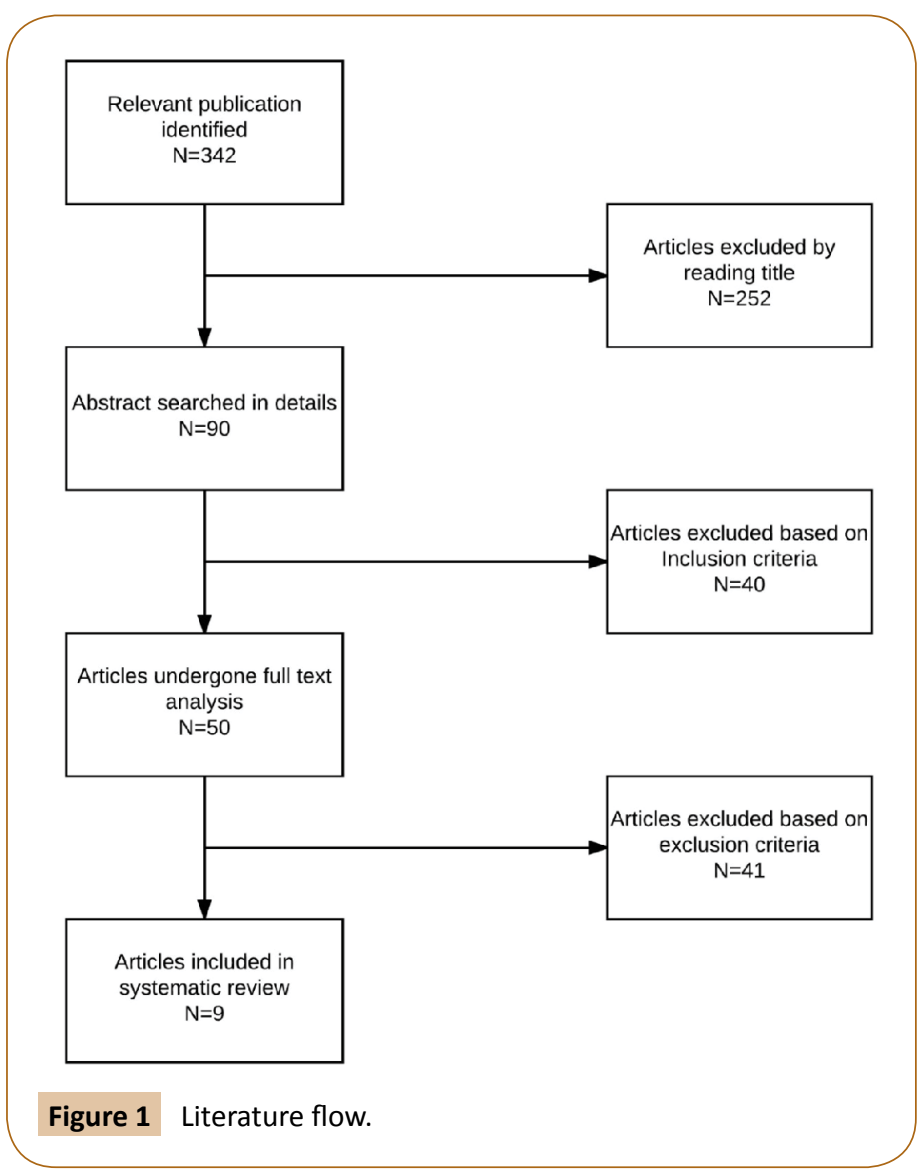


which 50 full text articles were examined in detail. In final nine studies were taken for final reviewing and quality and quantity data extraction.

After selection, full text articles were downloaded and hard copies were made. A systematic data extraction regarding basic details about articles [3,4,15-21], their sampling details (Table 1), laser and its characteristics (Table 2 ) and clinical findings and outcome (Table 3 ) and statistical outcomes of study (Table 4) were done.

Finalized articles were thoroughly evaluated for their quality and potential risk for bias based on an evaluation adapted from Cericato et al. (Table 5) [22]. The questions used for evaluation are; Q1. The abstract clearly presents the study objective, methodology, results and conclusion, Q2. The study exposes objective clear and precisely. Q3. The ethical aspects of the research are cited in the text. Q4. The research design is described. Q5. The sample size calculation is reported. Q6. The eligibility (1 point) and exclusion (1 point) criteria are described. Q7. Control groups are used. Q8. The research design is adequate (randomization and blinding).
Q9. The statistical tests are described. Q10. The $p$ values are cited. Q11. The study exposes the results clear and precisely. Q12. The study limitations are discussed.

Based on this evaluation each article were classified as low quality (0-8 points), moderate quality (9-11 points) or high quality (12-15 points). Six studies were of high quality and 3 were of medium quality based on risk bias.

Evaluation was done based on criteria for assessing study quality from the center of Reviews and Disseminations in York, UK (Table 6) [23].

Strong evidence: Randomized controlled trial, prospective studies/ large study samples

Well-defined and adequate control group clearly defined and clinically relevant variables

Low dropout rate

Relevant statistical analysis

Table 1 Study details.

\begin{tabular}{|c|c|c|c|c|c|c|c|c|c|}
\hline $\begin{array}{c}\text { Author and } \\
\text { year }\end{array}$ & $\begin{array}{c}\text { Sample } \\
\text { size, gender }\end{array}$ & Age(years) & $\begin{array}{l}\text { Study design } \\
\text { and blinding }\end{array}$ & Malocclusion & $\begin{array}{c}\text { Starting time } \\
\text { of tooth } \\
\text { movement }\end{array}$ & Anchorage & $\begin{array}{l}\text { Follow up } \\
\text { period }\end{array}$ & Force used & $\begin{array}{l}\text { Method of } \\
\text { measuring }\end{array}$ \\
\hline Curz [3] & $\begin{array}{c}11 \\
\text { Gender not } \\
\text { mentioned }\end{array}$ & Dec-18 & $\begin{array}{c}\text { RCT } \\
\text { Split mouth } \\
\text { Not specified }\end{array}$ & $\begin{array}{c}\text { Lack of space, } \\
\text { bimaxillary } \\
\text { protrusion }\end{array}$ & $\begin{array}{c}\text { Not } \\
\text { mentioned }\end{array}$ & $\begin{array}{c}\text { Nance arch, } \\
\text { TPA }\end{array}$ & 2 months & $150 \mathrm{gm} / \mathrm{month}$ & $\begin{array}{l}\text { Digital } \\
\text { electronic } \\
\text { calliper (in } \\
\text { loco) }\end{array}$ \\
\hline $\begin{array}{c}\text { Limpanichkul } \\
{[4]}\end{array}$ & $\begin{array}{c}12 \\
\text { 4-Male } \\
\text { 8-Female }\end{array}$ & $20.11+/-3.4$ & $\begin{array}{c}\text { RCT } \\
\text { Split mouth } \\
\text { Double blind }\end{array}$ & $\begin{array}{c}\text { Not } \\
\text { mentioned }\end{array}$ & $\begin{array}{c}3 \text { months after } \\
\text { extraction }\end{array}$ & $\begin{array}{c}\text { Vertical loop } \\
\text { stops mesial } \\
\text { molar tube }\end{array}$ & 3 months & $150 \mathrm{gm} / \mathrm{month}$ & $\begin{array}{l}\text { Stereo } \\
\text { microscope }\end{array}$ \\
\hline Youssef [15] & $\begin{array}{c}15 \\
\text { Gender not } \\
\text { mentioned }\end{array}$ & $14-23$ & $\begin{array}{l}\text { Prospective } \\
\text { CCT } \\
\text { Split } \\
\text { mouth Not } \\
\text { mentioned }\end{array}$ & $\begin{array}{c}\text { Lack of space, } \\
\text { bimaxillary } \\
\text { protrusion }\end{array}$ & $\begin{array}{c}14 \text { days after } \\
\text { extraction }\end{array}$ & $\begin{array}{c}\text { Not } \\
\text { mentioned }\end{array}$ & 9 weeks & $150 \mathrm{gm} / 21$ day & $\begin{array}{c}\text { Digital } \\
\text { electronic } \\
\text { calliper (in } \\
\text { cast) }\end{array}$ \\
\hline Sousa [16] & $\begin{array}{l}10 \text { 6-Male } \\
\text { 4-Female }\end{array}$ & 13. ' 1 & $\begin{array}{l}\text { RCT Split } \\
\text { mouth Not } \\
\text { mentioned }\end{array}$ & $\begin{array}{c}\text { Lack of space, } \\
\text { bimaxillary } \\
\text { protrusion }\end{array}$ & $\begin{array}{c}3 \text { months after } \\
\text { extraction }\end{array}$ & $\begin{array}{c}\text { Not } \\
\text { mentioned }\end{array}$ & 4 months & $150 \mathrm{gm} / \mathrm{month}$ & $\begin{array}{c}\text { Geometric } \\
\text { studio } 5 \\
\text { software }\end{array}$ \\
\hline $\begin{array}{c}\text { Doshi Mehta } \\
\text { [17] }\end{array}$ & $\begin{array}{c}20 \\
\text { 8-Male } \\
\text { 12-Female }\end{array}$ & Dec-23 & $\begin{array}{c}\text { RCT } \\
\text { Split mouth } \\
\text { Single blind }\end{array}$ & $\begin{array}{c}\text { Not } \\
\text { mentioned }\end{array}$ & $\begin{array}{c}21 \text { days } \\
\text { after SS wire } \\
\text { placement }\end{array}$ & TPA & 4-5 months & $150 \mathrm{gm}$ & $\begin{array}{c}\text { Digital } \\
\text { electronic } \\
\text { calliper (in } \\
\text { cast) }\end{array}$ \\
\hline Kansal [18] & $\begin{array}{c}10 \\
\text { Gender not } \\
\text { mentioned }\end{array}$ & $\begin{array}{c}\text { Not } \\
\text { mentioned }\end{array}$ & $\begin{array}{l}\text { Prospective } \\
\text { CCT } \\
\text { Split mouth } \\
\text { Triple blind }\end{array}$ & $\begin{array}{c}\text { Lack of space, } \\
\text { protrusion }\end{array}$ & $\begin{array}{c}\text { Not } \\
\text { mentioned }\end{array}$ & TPA & 63 days & $\begin{array}{c}150 \mathrm{gm} \\
\text { activation/35th } \\
\text { day }\end{array}$ & $\begin{array}{l}\text { Digital } \\
\text { electronic } \\
\text { calliper (in } \\
\text { loco) }\end{array}$ \\
\hline Heravi [19] & $\begin{array}{c}20 \\
\text { 3-Male } \\
\text { 17-Female }\end{array}$ & $22.1+/-5.3$ & $\begin{array}{l}\text { RCT } \\
\text { Split mouth } \\
\text { Single blind }\end{array}$ & $\begin{array}{c}\text { Not } \\
\text { mentioned }\end{array}$ & $\begin{array}{c}3 \text { months after } \\
\text { appliance } \\
\text { placement }\end{array}$ & $\begin{array}{l}\text { Vertical loop } \\
\text { stops mesial } \\
\text { to molar } \\
\text { tube }\end{array}$ & 56 days & $\begin{array}{c}150 \mathrm{gm} \\
\text { activation/28th } \\
\text { day }\end{array}$ & $\begin{array}{c}\text { Smile } \\
\text { analyser } \\
\text { software }\end{array}$ \\
\hline Dalaie [20] & $\begin{array}{c}12 \\
\text { 3-Male } \\
\text { 17-Female }\end{array}$ & 20.1 & $\begin{array}{l}\text { Randomised } \\
\text { CCT Split } \\
\text { mouth } \\
\text { Double blind }\end{array}$ & $\begin{array}{c}\text { Not } \\
\text { mentioned }\end{array}$ & $\begin{array}{c}3 \text { months } \\
\text { after premolar } \\
\text { extraction }\end{array}$ & $\begin{array}{c}\text { Not } \\
\text { mentioned }\end{array}$ & 67 days & $\begin{array}{c}150 \mathrm{gm} \text { activation/ } \\
\text { month }\end{array}$ & $\begin{array}{l}\text { Digital } \\
\text { electronic } \\
\text { caliper (in } \\
\text { cast) }\end{array}$ \\
\hline Ureturk [21] & $\begin{array}{c}15 \\
\text { 7-Male } \\
\text { 8-Female }\end{array}$ & $16.2+/-1.32$ & $\begin{array}{c}\text { RCT } \\
\text { Split } \\
\text { mouth Not } \\
\text { mentioned }\end{array}$ & $\begin{array}{l}\text { Angles class II } \\
\text { malocclusion }\end{array}$ & $\begin{array}{c}2 \text { weeks after } \\
\text { extraction }\end{array}$ & Mini implant & 90 days & $\begin{array}{c}150 \text { gm activation } \\
\text { done on } \\
21,42,63,84 \text { days }\end{array}$ & $\begin{array}{c}\text { Ortho } \\
\text { analyser } \\
\text { soft ware }\end{array}$ \\
\hline
\end{tabular}


Table 2 Details of laser used.

\begin{tabular}{|c|c|c|c|c|c|c|c|}
\hline Author and year & Type of laser & Wave length & $\begin{array}{l}\text { Energy } \\
\text { density }\end{array}$ & $\begin{array}{l}\text { Power } \\
\text { output }\end{array}$ & Time/tooth & $\begin{array}{l}\text { Points } \\
\text { irradiated }\end{array}$ & $\begin{array}{l}\text { Accelerated tooth } \\
\text { movement }\end{array}$ \\
\hline Curz [3] & Ga-Al-As diode laser & $780 \mathrm{~nm}$ & $5 \mathrm{~J} / \mathrm{sq} . \mathrm{cm}$ & $20 \mathrm{~mW}$ & $100 \mathrm{~s}$ & 10 & Yes \\
\hline Limpanichkul [4] & Ga-Al-As diode laser & $860 \mathrm{~nm}$ & $25 \mathrm{~J} / \mathrm{sq} . \mathrm{cm}$ & $100 \mathrm{~mW}$ & $184 \mathrm{~s}$ & 8 & No \\
\hline Youssef [15] & Ga-Al-As diode laser & $809 \mathrm{~nm}$ & $8 \mathrm{~J} / \mathrm{sq} \cdot \mathrm{cm}$ & $100 \mathrm{~mW}$ & $80 \mathrm{~s}$ & 6 & Yes \\
\hline Sousa [16] & Ga-Al-As diode laser & $780 \mathrm{~nm}$ & $5 \mathrm{~J} / \mathrm{sq} . \mathrm{cm}$ & $20 \mathrm{~mW}$ & $100 \mathrm{~s}$ & 10 & Yes \\
\hline Doshi Mehta [17] & Ga-Al-As diode laser & $810 \mathrm{~nm}$ & $8 \mathrm{~J} / \mathrm{sq} . \mathrm{cm}$ & $100 \mathrm{~mW}$ & $100 \mathrm{~s}$ & 10 & yes \\
\hline Kansal [18] & Ga-As diode laser & 904 nm & $4.2 \mathrm{~J} / \mathrm{sq} . \mathrm{cm}$ & $12 \mathrm{~mW}$ & $100 \mathrm{~s}$ & 10 & Yes \\
\hline Heravi [19] & Ga-Al-As diode laser & $810 \mathrm{~nm}$ & $21.4 \mathrm{~J} / \mathrm{sq} . \mathrm{cm}$ & $200 \mathrm{~mW}$ & $300 \mathrm{~s}$ & 10 & No \\
\hline Dalaie [20] & Ga-Al-As diode laser & $800 \mathrm{~nm}$ & $5 \mathrm{~J} / \mathrm{sq} . \mathrm{cm}$ & $100 \mathrm{~mW}$ & $80 \mathrm{~s}$ & 8 & No \\
\hline Ureturk [21] & Ga-Al-As diode laser & $820 \mathrm{~nm}$ & $5 \mathrm{~J} / \mathrm{sq} \cdot \mathrm{cm}$ & $20 \mathrm{~mW}$ & $100 \mathrm{~s}$ & 10 & Yes \\
\hline
\end{tabular}

Table 3 Frequency of laser application and rate of tooth movement.

\begin{tabular}{|c|c|c|c|c|c|c|c|c|c|}
\hline \multirow{3}{*}{ Author and year } & \multirow{3}{*}{$\begin{array}{l}\text { Frequency of application } \\
\text { (days) }\end{array}$} & \multicolumn{8}{|c|}{ Orthodontic tooth movement rate (in $\mathrm{mm}$ ) } \\
\hline & & \multicolumn{2}{|c|}{ 1st month } & \multicolumn{2}{|c|}{ 2nd month } & \multicolumn{2}{|c|}{ 3rd month } & \multicolumn{2}{|c|}{ 4-5th month } \\
\hline & & control & experiment & control & Experiment & control & experiment & control & Experiment \\
\hline Curz [3] & $0,3,7,14,30,33,37,44$ & & & $33.30+/-0.24$ & $4.39+/-0.27$ & & & & \\
\hline Limpanichkul [4] & $1,2,3$ day of every activation & 0.38 & 0.32 & 0.74 & 0.73 & 1.24 & 1.29 & & \\
\hline \multirow[t]{2}{*}{ Youssef [15] } & $0,3,7,14$ day of every activation & \multicolumn{2}{|c|}{ Mean for 9 weeks } & & & & & & \\
\hline & & 1.019 & 2.027 & & & & & & \\
\hline Sousa [16] & $0,3,7$ day of activation & $0.42+/-0.29$ & $1.16+/-0.51$ & $0.80+/-0.49$ & $2.05+/-0.93$ & $1.60+/-0.63$ & $3.09+/-1.06$ & & \\
\hline \multirow[t]{3}{*}{ Doshi Mehta [17] } & $0,3,7,14,45,75,105,135$ & & & & & & & & \\
\hline & Maxilla & & & & & $0.66+/-0.55$ & $1.43+/-0.15$ & $\begin{array}{c}0.84+/- \\
0.21\end{array}$ & $1.17+/-0.22$ \\
\hline & Mandible & & & & & $0.35+/-0.28$ & $1.51+/-0.18$ & $\begin{array}{c}0.75+/- \\
0.09\end{array}$ & $1.11+/-0.17$ \\
\hline Kansal [18] & $0,3,7,14,21,28,35,42,49,56$ & $1.76+/-1.58$ & $1.68+/-1.20$ & $3.30+/-2.36$ & $3.53+/-2.30$ & & & & \\
\hline \multirow[t]{2}{*}{ Heravi [19] } & $3,7,11,15$ & \multicolumn{2}{|c|}{ Mean for 56 days } & & & & & & \\
\hline & & $2.11+/-1.14$ & 2.13 & $/-1.16$ & & & & & \\
\hline \multirow[t]{3}{*}{ Dalaie [20] } & Single exposure & & & & & & & & \\
\hline & Maxilla & $2.49+/-0.98$ & $2.61+/-1.59$ & $4.5+1-0.23$ & $4.98+/-0.78$ & & & & \\
\hline & Mandible & $2.03+/-1.56$ & $2,29+/-1.36$ & $4.01+/-1.44$ & $3.73+/-1.08$ & & & & \\
\hline \multirow[t]{2}{*}{ Ureturk [21] } & $\begin{array}{c}0,3,7,14,21,30,33,37,60,63 \\
67,74,81,84,90 \text { days }\end{array}$ & \multicolumn{2}{|c|}{ Movement for 3 months } & & & & & & \\
\hline & & \multicolumn{2}{|c|}{$2.77+/-1.49$} & $3.9+/-1.41$ & & & & & \\
\hline
\end{tabular}

Table 4 Clinical and statistical out comes and evidence grading.

\begin{tabular}{|c|c|c|c|c|}
\hline Author and year & Clinical outcome & Pvalue & Statistical significance & Evidence grading \\
\hline Curz [3] & $34 \%$ more movement on experimental side & $<0.001$ & Highly significant & A \\
\hline Limpanichkul [4] & No difference between both sides & 0.77 & Non-significant & A \\
\hline Youssef [15] & 1.98 fold more in laser group & $<0.05$ & Significant & B \\
\hline Sousa [16] & Greater movement on laser side & 0.0001 to 0.029 & Highly significant & A \\
\hline Doshi Mehta [17] & $30 \%$ more on laser side & 0.000 to 0.0381 & Highly significant & A \\
\hline Kansal [18] & Faster in laser side & 0.34 to 0.69 & Non-significant & A \\
\hline Heravi [19] & No difference between both sides & $>0.05$ & Non-significant & B \\
\hline Dalaie [20] & No difference between both sides & 0.45 & Non-significant & B \\
\hline Ureturk [21] & $40 \%$ faster on laser side & 0.001 & Highly significant & $A$ \\
\hline
\end{tabular}

Moderately strong evidence: Prospective study, cohort, controlled clinical trial, or well-defined retrospective study with large study group

Clearly defined and clinically relevant variables

Low dropout rate

Relevant statistical analysis

Limited evidence: Cross-sectional study
Clinically inadequate result variables

High dropout rate

No control group of its own in the study Limited/no statistical analysis

Addressing the issue in question only in part.

\section{Results}

Out of the nine studies which were evaluated for evidence, six 
Table 5 Potential risk for bias (Cericato et al).

\begin{tabular}{|c|c|c|c|c|c|c|c|c|c|c|c|c|c|c|}
\hline Author and year & $\begin{array}{l}\text { Q.1 (1 } \\
\text { point) }\end{array}$ & $\begin{array}{l}\text { Q.2 (1 } \\
\text { point) }\end{array}$ & $\begin{array}{l}\text { Q.3 (1 } \\
\text { point) }\end{array}$ & $\begin{array}{l}\text { Q.4 (1 } \\
\text { point) }\end{array}$ & $\begin{array}{l}\text { Q.5(2 } \\
\text { points) }\end{array}$ & $\begin{array}{l}\text { Q.6 (2 } \\
\text { points) }\end{array}$ & $\begin{array}{l}\text { Q.7 (1 } \\
\text { point) }\end{array}$ & $\begin{array}{l}\text { Q.8 (2 } \\
\text { points) }\end{array}$ & $\begin{array}{l}\text { Q.9 (1 } \\
\text { point) }\end{array}$ & $\begin{array}{l}\text { Q.10 (1 } \\
\text { point) }\end{array}$ & $\begin{array}{l}\text { Q.11 (1 } \\
\text { point) }\end{array}$ & $\begin{array}{l}\text { Q.12 (1 } \\
\text { point) }\end{array}$ & $\begin{array}{c}\text { Overall } \\
\text { score }\end{array}$ & Quality \\
\hline Curz [3] & 1 & 1 & 1 & 1 & 0 & 1 & 1 & 0 & 1 & 1 & 1 & 0 & 9 & Moderate \\
\hline Limpanichkul [4] & 1 & 1 & 0 & 1 & 0 & 1 & 1 & 2 & 1 & 1 & 1 & 1 & 11 & Moderate \\
\hline Youssef [15] & 1 & 1 & 1 & 1 & 0 & 2 & 1 & 1 & 1 & 1 & 1 & 1 & 12 & high \\
\hline Sousa [16] & 1 & 1 & 1 & 1 & 0 & 1 & 1 & 2 & 1 & 1 & 1 & 1 & 12 & High \\
\hline Doshi Mehta [17] & 1 & 1 & 1 & 1 & 2 & 1 & 1 & 2 & 1 & 1 & 1 & 0 & 13 & High \\
\hline Kansal [18] & 1 & 1 & 1 & 1 & 0 & 1 & 1 & 2 & 1 & 1 & 1 & 1 & 12 & High \\
\hline Heravi [19] & 1 & 1 & 1 & 1 & 0 & 1 & 1 & 1 & 1 & 1 & 1 & 1 & 11 & Moderate \\
\hline Dalaie [20] & 1 & 1 & 1 & 1 & 0 & 2 & 1 & 2 & 1 & 1 & 1 & 1 & 13 & High \\
\hline Ureturk [21] & 1 & 1 & 1 & 1 & 0 & 1 & 1 & 2 & 1 & 1 & 1 & 1 & 12 & High \\
\hline
\end{tabular}

Table 6 Evaluation for conclusion.

\begin{tabular}{|cc|}
\hline Grade of evidence & Criteria \\
\hline Grade 1 evidence & Two studies with strong evidence \\
\hline Grade 2 evidence & One study with strong evidence and two studies with moderate evidence \\
\hline Grade 3 evidence & Two studies with moderate evidence \\
\hline Grade 4 evidence & Insufficient scientific support \\
\hline
\end{tabular}

Table 7 Possible reasons for negative results.

\begin{tabular}{cc}
\hline Article & Possible reasons for negative results \\
\hline Limpanichkul [3] & High energy density used \\
\hline Kansal [18] & Ga-Ar diode laser used \\
\hline Heravi [19] & High power output used \\
\hline Dalaie [20] & Reduced frequency of exposure
\end{tabular}

were of high evidence and three were of moderate evidence. Out of the six high evidence studies four showed statistically highly significant result and two showed non-significant result. Out of the three medium evidence studies two showed statistically nonsignificant result and one showed significant result. So in effect there is some evidence for LLLT causing accelerated orthodontic tooth movement. The results of evaluation are given in Table 4.

\section{Discussion}

From result it is evident that four of high evidence studies and one of moderate evidence study favours accelerated orthodontic tooth movement. On the other hand two of high evidence and two of moderate evidence studies showed non-significant results.

All the four studies which showed non-significant results had a mildly faster tooth movement on experimental side during some period of study.

Studies which failed to demonstrate clinical acceleration in tooth movement had clearly mentioned some factors that might have caused an alteration from the generalized trend of positively accelerated tooth movement Table 7.

Conclusion of effect of LLLT on accelerated orthodontic tooth movement was drawn based on the criteria for assessing study quality from the centre of Reviews and Disseminations in York, UK [23] . Comparing the parameters of laser used in different studies, all studies except one study [18] used Ga-Al-As laser in continuous wave mode and all of them fall at a wavelength of 780-904 $\mathrm{nm}$ which are infra-red in nature which have low absorption coefficient with haemoglobin and water so high penetration depth in irradiated area [16] .

From Table 2, on considering the energy density, $4.2-8 \mathrm{~J} / \mathrm{sq} . \mathrm{cm}$ produced a favourable effect, were as a high value of $25 \mathrm{~J} /$ sq.cm and $2.14 \mathrm{~J} / \mathrm{sq} . \mathrm{cm}$ couldn't accelerate tooth movement. It was also observed that based on power output, 20-100 mW gave a positive result whereas $200 \mathrm{~mW}$ gave no effect on tooth movement which points that very high power output could be the reason of negative outcome. According to Yamagashi [24] approximately $50 \%$ of laser penetrates to a depth of $1 \mathrm{~mm}$ in human cortical bone at a power of $60 \mathrm{~mW}$. This explains why the studies used the range of $20-100 \mathrm{~mW}$ of power with tissue contact in order to minimise reflection of laser giving positive result.

As far as number of irradiation points are concerned all of studies used 6-10 irradiation points based on root morphology, which points that every study needed distribution of total exposure rather than energy concentrating on certain areas. Irradiation intervals also varied in different studies. Studies with positive result had a range of 4-6 exposures per month whereas study with one exposure and three exposures failed to give positive result (Table 3).

Rate of tooth movement was found to be maximum during 3-4 months of irradiation [16,17] even $30 \%$ faster movement was noted [17].

Another thing to be considered is the systemic effect of phototherapy, if proper shielding is not used in split mouth 
studies chances for irradiation of control sites can give faulty readings. No studies mentioned this. Even if the laser effect is controversial in some studies, it is clear that these controversies might be the result of differences in study design, laser variables applied and sample size. Random variables and numerous factors interacting with tooth movement clinically also plays a role in it. Still it remains a question which combination of laser parameters and exposure yields in productive accelerated tooth movement. Hence more studies with adequate sample size are required to set a standard protocol. Excluding the study used laser other than Ga-Al-As, we find a strong evidence of accelerated tooth movement (Table 2).

The bone density varies between maxilla and mandible also between different regions of jaw, so tooth movement at other regions might be a little different from canine even if same dosimetry and laser parameters considered. Hence studies with canine movement were only included in our inclusion criteria. There was a trend of increased maxillary and decreased

\section{References}

1 Tsichlaki A, Chin SY, Pandis N, Fleming PS (2016) How long does treatment with fixed orthodontic appliances last? A systematic review. Am J Orthod Dentofacial Orthop 149: 308-318.

2 Segal GR, Schiffman PH, Tuncay OC (2004) Meta-analysis of the treatment related factors of external apical root resorption. Orthod Craniofac Res 7: 71-78.

3 Cruz DR, Kohara EK, Ribeiro MS, Wetter NU (2004) Effects of low intensity laser therapy on the orthodontic movement velocity of human teeth: a preliminary study. Lasers Surg Med 35: 117-120.

4 Limpanichkul W, Godfrey K, Srisuk N, Rattanayatikul C (2006) Effects of low level laser therapy on the rate of orthodontic tooth movement. Orthod Craniofac Res 9: 38-43.

5 Patterson BM, Dalci O, Darendeliler MA, Papadopoulou AK. Corticotomies and orthodontic tooth movement: a systematic review. J Oral Maxillofac Surg 74: 453-473.

6 Hoogeveen EJ, Jansma J, Ren Y (2014) Surgically facilitated orthodontic treatment: a systematic review. Am J Orthod Dentofac Orthop145: S51-64.

7 Miles P (2017) Accelerated orthodontic treatment what's the evidence? Aus Dent J 62: 63-70.

8 Harradine NW (2001) Self-ligating brackets and treatment efficiency. J Clin Orthod Res 4: 220-227.

9 Madan MS, Liu ZJ, Gu GM, King GJ (2007) Effects of human relaxin on orthodontic tooth movement and periodontal ligaments in rats. Am J Orthod Dentofac Orthop 131: e1-e10.

10 Nambi N, Shrinivaasan NR, Dhayananth LX, Chajallani VG, George AM (2016) Renaissance in orthodontics: Nanotechnology. Int J Orthod Rehabil 7: 139-143.

11 Domínguez A, Gómez C, Palma JC (2015) Effects of low-level laser therapy on orthodontics: rate of tooth movement, pain, and release of RANKL and OPG in GCF. Lasers Med Sci 30: 915-923.

12 Genc G, Kocadereli I, Tasar F, Kilinc K, El S, et al. (2013) Effect of lowlevel laser therapy (LLLT) on orthodontic tooth movement. Lasers Med Sci 28: 41-47. mandibular tooth movement towards third and fourth month of laser exposure, which may be due to variations in bone density and vasculature.

Some systematic reviews and meta-analysis $[25,26]$ concluded that there is no evidence for LLLT having effect on accelerated orthodontic tooth movement. But they considered small number of studies only. Hence this review tried the best to include latest studies and re-evaluate the effect of LLLT. Incomparable heterogeneity in extracted data was the biggest problem we faced. Lack of large sampled studies with long term evaluation is the drawback of this review.

\section{Conclusion}

There is some evidence of accelerated orthodontic tooth movement with low level laser therapy. It is a promissory procedure with a potential to accelerate tooth movement. More high evidence studies are required for concrete conclusion.

13 Liberati A, Altman DG, Tetzlaff J, Mulrow C, Gøtzsche PC, et al. (2009) The PRISMA statement for reporting systematic reviews and meta-analyses of studies that evaluate health care interventions: explanation and elaboration. J Clin Epidemiol 62: e1-34.

14 Jain S, Sharma N, Jain D (2015) Basic fundaments of designing a quality research. J Adv Med Dent Sci Res 3: 88-95.

15 Youssef M, Ashkar S, Hamade E, Gutknecht N, Lampert F, et al. (2008 )The effect of low-level laser therapy during orthodontic movement: a preliminary study. Lasers Med Sci 23: 27-33.

16 da Silva Sousa MV, Scanavini MA, Sannomiya EK, Velasco LG, Angelieri $F$ (2011) Influence of low-level laser on the speed of orthodontic movement. Photomed Laser Surg 29: 191-196.

17 Doshi-Mehta G, Bhad-Patil WA (2012) Efficacy of low-intensity laser therapy in reducing treatment time and orthodontic pain: a clinical investigation. Am J Orthod Dentofac Orthop 141: 289-297.

18 Kansal A, Kittur N, Kumbhojkar V, Keluskar KM, Dahiya P (2014) Effects of low-intensity laser therapy on the rate of orthodontic tooth movement: a clinical trial. Dent Res J 11: 481-488.

19 Heravi F, Moradi A, Ahrari F (2014)The effect of low level laser therapy on the rate of tooth movement and pain perception during canine retraction. Oral Health Dent Manag 13: 183-188.

20 Dalaie K, Hamedi R, Kharazifard MJ, Mahdian M, Bayat M (2015) Effect of low-level laser therapy on orthodontic tooth movement: a clinical investigation. J Dent 12: 249-256.

21 Üretürk SE, Saraç M, Fıratlı S, Can ŞB, Güven Y, et al. (2017) The effect of low-level laser therapy on tooth movement during canine distalization. Lasers Med Sci 32: 757-764.

22 Cericato GO, Bittencourt MA, Paranhos LR (2015) Validity of the assessment method of skeletal maturation by cervical vertebrae: a systematic review and meta-analysis. Dentomaxillofac Radiol 44 20140270.

23 Khan KS, Ter Riet G, Glanville J, Sowden AJ, Kleijnen J (2001) Undertaking systematic reviews of research on effectiveness: CRD's guidance for carrying out or commissioning reviews. Research Report. CRD Report 4 (2n). NHS Centre for Reviews and Dissemination, York, UK.

24 Yamagishi H, Shinohara C, Saito S, Sasaki H, Kanegae H, et al. (1994) 
A basic study on the use of semiconductor laser of penetrative sensitivity on living tissue. J Jpn Soc Laser Dent 5: 13-22.

25 Long H, Pyakurel U, Wang Y, Liao L, Zhou Y, et al. ( 2012) Interventions for accelerating orthodontic tooth movement: a systematic review. Angle Orthod 83: 164-171
26 de Almeida VL, de Andrade Gois VL, Andrade RN, Cesar CP, de Albuquerque-Junior RL, et al. (2016) Efficiency of low-level laser therapy within induced dental movement: a systematic review and meta-analysis. J Photochem Photobiol B: Biol 158: 258-266. 